\title{
Conceptual Framework Based On Type-2 Fuzzy Logic Theory for Predicting Childhood Obesity Risk
}

\author{
https://doi.org/10.3991/ijoe.v16i03.12701 \\ Khalid Almohammadi \\ University of Tabuk, Tabuk, Saudi Arabia \\ kalmohammadi@ut.edu.sa
}

\begin{abstract}
Obesity is a critical public health concern affecting a wide range of people globally. The rise in obesity is limited to not only the wealthiest countries but also the poorest. Childhood obesity has grown exponentially in the last few years, and its progression is significant contribution to the increase in mortality rates. Childhood obesity is linked with a wide range of risk factors. These include individual and parental biological factors, sedentary behavior or decreased physical activity, and parent restriction. This paper focuses on reviewing the techniques of artificial intelligence (AI) utilized in the management of obesity in children. The paper will also propose a conceptual framework based on a novel type-2 fuzzy logic methodology, which can predict risks for developing childhood obesity. The proposed approach will address factors such as family characteristics, unhealthy food choices and lack of exercise, and others related to children and their home environment. The procedure will help in the prevention of childhood obesity, promote public health, and reduce treatment costs for a wide range of obesity-related conditions. The proposed conceptual framework will be evaluated using type- 2 fuzzy logic systems on approximately one thousand families in Saudi Arabia and their children. This proposed system will be able to handle the encountered uncertainties to enhance modeling and promote the accuracy of predictions of the risk for childhood obesity. This system can also encode extracted rules comprehensively to provide insight into the best childhood obesity prevention behaviors.
\end{abstract}

Keywords - Childhood obesity, type-2 fuzzy logic, data-driven approach

\section{Introduction}

In the contemporary global community, obesity is considered among the most prevalent dietary issues in both developed and developing nations. The high prevalence of childhood obesity has attracted the attention of relevant stakeholders such as parents, clinicians, advocates of the pediatric population, and policy makers [1], [2]. The concern of these stakeholders is that the children who are overweight and obese will become adults who have the potential to be obese and overweight [3]. Pediatric obesity has significant consequences relating to psychosocial and physical health status. Accordingly, most of the heart-related impacts of adult-onset obesity can be traced back to childhood abnormalities [4], [5]. Cases of hypertension, abnormal glu- 
cose tolerance, and hyperlipidemia are prevalent among the obese pediatric population. Additionally, obesity has been linked to long-term adverse impacts, resulting in asthma, nonalcoholic steatohepatitis, hypertrophy of the left ventricle, sleep apnea, orthopedic complications, and psychological concerns [4]. Despite the vast nature of developmental and clinical challenges facing children with obesity, they are usually underrated.

Considering the substantial consequences of obesity, the prevention of this condition among the pediatric population is a global public-health prime concern [7]. Presently the prevention approaches implemented among children have the potential to improve behaviors that are risk factors for obesity [8]. Nonetheless, the efficacy of these strategies in obesity prevention has not been explored [8]. Parents dictate the genetic makeup and surroundings of children [9]. These two factors are crucial in developing children's dietary habits and influencing weight gain, especially in the first years of their lives [9]. It is essential to note that there are other environmental factors that a child may be exposed to before and after birth. These risk factors have a significant foundation in the home and family surroundings [9]. Furthermore, they may be diverse in terms of their level of modifiability and availability of evidence supporting their association with obesity development among the pediatric population [9].

One of the limitations of prospective cohort and epidemiological research is that it has the potential to determine participants and groups at high risk but cannot provide evidence for cause-and-effect relationships [9]. Therefore, existing epidemiological studies are vulnerable to spurious correlation, while practice based on this evidence is susceptible to failure due to lack of an established association between the variables and clinical outcomes [9]. It is essential to determine causal evidence from studies to develop effective prevention strategies. To develop effective obesity prevention strategies, a cause-and-effect association between obesity-related variables should be established.

Many intervention and prevention strategies have been proposed; however, they are fashioned only for preschoolers as opposed to the entire pediatric population [10]. Therefore, primary prevention strategies for pediatric obesity need to be extended to cover other factors, settings, and developmental stages [10]. For instance, young adults find it challenging to maintain a healthy weight as a result of lifestyle changes linked to adulthood, such as work, independent living, and starting a family [9]. Implementation of prevention strategies is essential in the early developmental stages of children and in transitioning to parenthood of young adults, respectively. Another notable limitation of existing prevention and intervention is that they are founded on the process of prior theoretical framework with one model fits all.

The contemporary scientific field is undergoing a vital transformation, changing from a prior framework to a data-driven posterior framework [11]. One of the examples of the data-driven posterior framework is the computational-centric study [11]. A computational-centric study refers to an approach of data exploration that amalgamates the first three approaches of theory, experimentation, and simulation. The fourth methodological shift postulates a different era of scientific studies that are focused on computational processing of data and storage independent of human intervention [12]. 
Artificial Intelligence (AI), which is the basis for developing advanced technology, has been characterized as disruptive in the clinical setting and is anticipated to aid healthcare specialists in using extracted data and transforming data into useful realtime health information by 2025 [11], [13], [14]. Furthermore, 90 percent of US studies and 60 percent of international clinical and insurance organizations are expected to utilize AI [11], [13]. Some of the factors facilitating the implementation of AI in this sector are decreased healthcare costs, patient-centric intervention plans, and amelioration of patient outcomes. As such, technological applications in the healthcare sector can be significantly improved by shifting from fixed pre-instructed software to selflearned systems using machine learning (ML). With ML-based systems, effective obesity prevention approaches can be continuously improved [11], [14].

This paper presents a review of the AI and data-driven approaches for preventing and predicting childhood obesity. In addition, it proposes conceptual frameworks that aim to use a type-2 fuzzy logic methodology, which can predict the risk of obesity for children on their family's dirty habits patterns, characteristics, and other parameters. This prediction will be used as an intervention factor to remediate obesity, which will enhance public health and reduce the costs of later treatments for several obesityrelated diseases. We will conduct the assessment of the proposed system on at least one thousand families in Saudi Arabia. The proposed type-2 fuzzy logic systems will be capable of handling the encountered uncertainties to achieve better modeling and more accurate results on the risk for obesity in children. it can also encode the extracted rules in comprehensive ways to provide insights into the best obesity prevention behaviors for lowering childhood obesity risk.

This paper is divided into five sections: section 2 reviews the AI and data-driven approaches used for childhood obesity; section 3 describes the conceptual framework based on type- 2 fuzzy logic systems; section 4 explains the primary setup of experimental design and methodology; and section 5 comprises the conclusion and recommendations for future works.

\section{Review of AI and Data-Driven Approaches Used for Childhood Obesity}

Most AI and ML techniques are now widely used and considered to be promising tools in the revolutions of various fields such as medicine and nutrition science. Many studies have implemented AI and ML to assess obesity risk in adulthood with little consideration to preventing it; however, the prediction model could be beneficial for educating people about whether they fall into the clinical categories or parameters for overweight and obesity. Neural networks, along with parameter-decreasing methods (PDM), have been used to diagnose the variables that include the start of obesity to predict the increase in body mass index (BMI) [15]. Fuzzy logic has been used in predicting obesity levels to guide specialists in the decision-making process for enhancing patient welfare and minimizing morbimortality [16]. Cruz and his colleagues used a Bayesian network to develop the decision support system to assist nutrition specialists in the nutrition diagnoses of bariatric surgery patients [17]. 
In one study [18], a support-vector machine was used to survey feeding activities in a sample of overweight participants based on their swallowing and chewing habits. The model achieved 94 percent accuracy when the swallowing sequence and chewing-signals information were measured. K-means clustering, an unsupervised form of ML, was utilized in the study of obesity and its intervention. The K-means technique was crucial in classifying weight dynamics among participants of the study. It is important to note that healthcare professionals often have a standard threshold for weight change.

Notwithstanding possible current guidelines, using a data-driven approach, an analytical weight-change threshold was determined. This study classification was done with the strategic objective of finding the weight-change limit [19]. The K-means approach has been critical in determining and categorizing weight change for subjects in dietary- and weight-intervention projects. According to the conducted experiment, the K-means clustering technique yielded a relatively more accurate set of thresholds that can be integrated with clinicians' knowledge in the classification of weightchange performance for participants of a dietary/weight-centric intervention program [19].

Various studies have been conducted using ML techniques to predict childhood and adolescent obesity and link them to the related causation variables. A few reviews exist to predict childhood obesity based on ML techniques. Some of the studies elaborate effectively on the algorithms used but do not have factual experimental findings [20],[21],[22]. Another shortcoming of the existing evidence is the participants' ages. In one study [23], the data used were from children with an age range of nine to eleven. This age range is not adequate for predicting obesity because within this age range obesity usually has established itself, thus making it pointless to apply predictive and preventive initiatives. Apart from this issue, the studies are also faced with samplesize limitations. For instance, one investigation that aimed to apply ML and AI in pediatric obesity prediction used data from subjects with specific behaviors and small samples of twelve participants [24].

Although existing evidence related to the use of AI and ML in predicting pediatric obesity has several limitations, better studies addressing this research topic also exist. Accordingly, Zhang et al. conducted research and presented a comprehensive analysis of ML approaches to forecasting pediatric obesity [25]. In this research, the authors compared the performance measures of various ML approaches. The study found that optimum sensitivity - which is critical in predicting obesity - for all ML techniques was 62 percent and was achieved when predicting obesity of subjects more than one year old. Nonetheless, the study does not provide estimates of specificity and accuracy. In addition, although the study was conducted with the aim of predicting pediatric obesity for children more than one year old, the data used from a clinical decision support system were for participants less than one year old [26].

Most of the approach studies used are black box [26], [27], [28], [29], [30], which means that parents or specialists cannot understand the intervention information. This does not encourage parents to contribute to the intervention's success. The majority of the algorithms - for example, neural networks, hidden Bayesian networks and Markov models, and neural networks - cannot relay transparent information about human 
behavior and habits and their related outputs. Therefore, the lack of clarity of the results and their interpretation [31], [32] creates great difficulty for users. Studies conducted on childhood and adolescent obesity [26], [27], [28], [29], [30] using the black-box approach are also limited, because researchers should do iterative long-time learning procedures to fit their models according to the dynamic nature of human behaviors.

Fuzzy systems will have a significant impact on predicting the obesity of children because of their capabilities to extract easy-to-read models from the learned models in the intervention process and give parents insight into the success of intervention behaviors. Type-2 fuzzy logic systems have a greater degree of freedom because of their footprint of uncertainty in the third dimension of type-2 fuzzy sets. This indicates that owing to their type-2 fuzzy sets, type- 2 fuzzy logic approaches perform better than type-1 [33], [34]. The type-2 fuzzy logic system provides a strategy for solving various sources of uncertainty in diagnosing obesity and its associated behaviors. To the best of the authors' knowledge, no studies have applied type- 2 fuzzy logic to predict childhood obesity. So, we hypothesis type-2 fuzzy logic systems can handle the uncertainties regarding modeling, prediction, and encoding of the extracted rules in a comprehensive way to yield insight into the best obesity prevention behaviors.

\section{The Conceptual Framework Based on a Novel Type-2 Fuzzy Logic System for Predicting Childhood Obesity Risk}

The aim of the proposed conceptual type-2 fuzzy logic is to gain insight and knowledge regarding several childhood obesity-caused parameters that are related to children themselves and the impact of their parents-such as parents' educational level, genetic background, dietary patterns, and other factors-along with physical activity level, which has the ability to affect children's BMI. The ability of fuzzy logic to reason with imprecise and uncertain data in addressing the problem of diagnosis as well as monitoring diseases cannot be overemphasized [35]. The choice of type-2 fuzzy logic-based systems is attributed to the fact that they offer better performance for problems or issues that involve a level of uncertainty. Although type-2 fuzzy logic has been applied in many areas, there is little understanding about its applicability in predicting obesity. The proposed conceptual model contains the following three components (see Fig. 1):

- Causes of Childhood Obesity Component (CCOC)

- Intelligent-Data Driven Learning Component (IDDLC)

- The Predictor of Childhood Obesity Risk and Lifelong Learning Component (PCORLLC) 


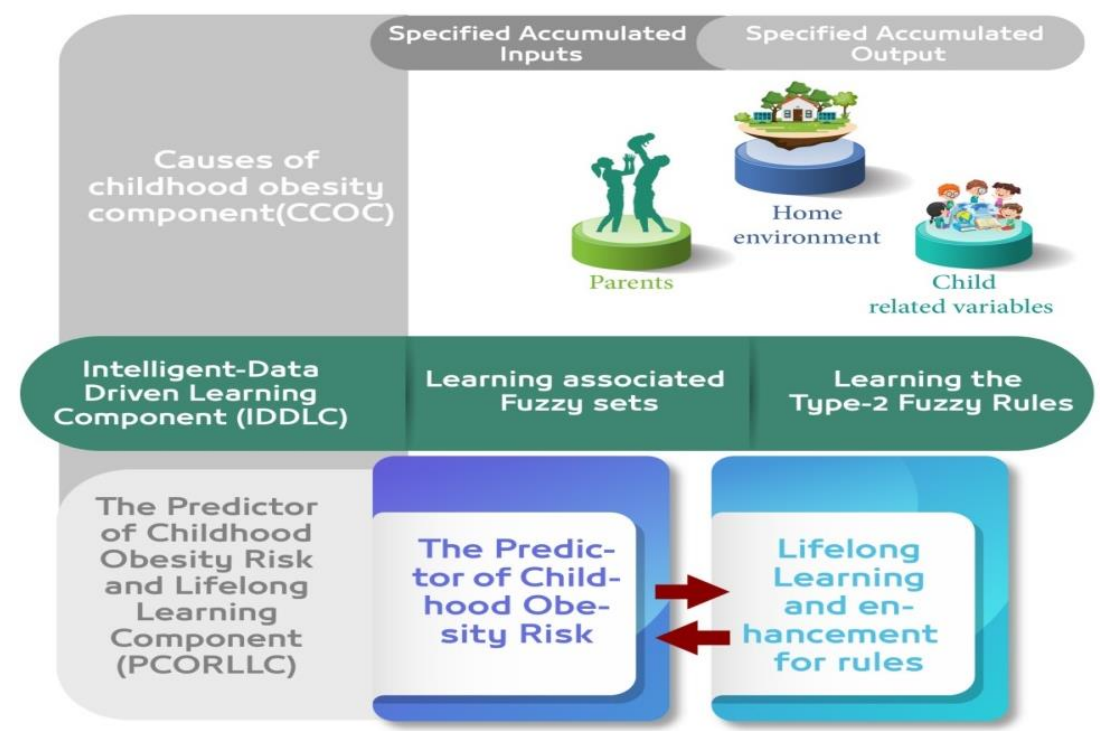

Fig. 1. The Conceptual model based on type-2 fuzzy logic theory for predicting childhood obesity risk

The proposed conceptual framework will begin working at the causes of childhood obesity component (CCOC), where the child interacts with his or her environment and parents. First, this component is dedicated to specifying the required childhood obesity-caused parameters needed to learn the impact of the home environment on the childhood risk for obesity. The environment is a crucial factor in the development of childhood obesity. Therefore, measuring what and whom children interact with will help identify the relationship between the environment and obesity. Next, this component will gather and record the collected data concerning childhood obesity (the model inputs and outputs), helping establish the link between inputs and outputs. The intelligent-data driven learning component (IDDLC) will extract the model-learned rules after receiving the inputs and outputs from the CCOC.

This component will learn three type-2 fuzzy sets (based on a sample of the opinions of twenty experts in the field of childhood obesity) using the methodology described in [28], [29], [30], [31] to handle linguistic uncertainty. After completing the workflow from the previous components, the proposed model will then "learn-build" the fuzzy rules that can predict the risk for childhood obesity with regard to the current causes using the unsupervised approach detailed in [32], [33], [34]. The use of an intelligent system will help piece together information from various sources to make correct predictions about the risk that a child faces. Finally, the predictor of childhood obesity risk and lifelong-learning component (PCORLLC) will be utilized; this is where the child's current home environment, parents, and the child him- or herself input conditions that input previous and current conditions that predict the child's risk for obesity and provide insight into how to change the risk level. The proposed conceptual framework will facilitate real-time prediction, make improvements on the 
existing rules in this component, and learn from new cases. It incorporates a lifelonglearning mechanism to exhibit new behaviors that are collected from the experience of obesity in childhood. It will also ensure that changes are evidence based, with particular focus on the interactions that children have and on their exposure to elements that predispose them to obesity. It will help develop guidelines on the behaviors that ensure that children grow up healthy.

\section{Experimental Set-Up}

The study will rely on an experimental research format, a gold standard in study designs. It is applicable to studies in which the researcher needs to establish the connection between an independent variable and dependent ones. It is Somewhat useful for cause-and-effect relationships, where the results or the outcomes are observed based on the inputs. Experimental designs can be either true experimental or quasiexperimental. This research includes multiple disciplines, such as AI (ML), computer science, and nutrition. It will start by studying the factors affecting childhood obesity and related behavior as well as other associated factors that have an impact, such the home environment and the child him- or herself. Obesity tracks in families, and the strongest predictor of childhood obesity is the BMI of the father and mother. Children with low fitness and increased sedentary time in combination with decreased physical activity often have significantly higher risks for obesity. Family characteristics and dirty habit patterns, such as making poor dietary choices and engaging in sedentary behavior, play a significant role in the development of childhood obesity [36], [37]. These factors will form the cornerstone in the process of building the proposed systems, which will also be based on the related studies mentioned in the literature section. Afterward, this study will identify the variables in the home environment related to the rise in obesity and other variables that can be added and linked with the risk. Data collection will be based on the selected variables (an initial one is shown in table $1)$;

Table 1. Initial Selected Factors

\begin{tabular}{|c|l|}
\hline Factor number & \multicolumn{1}{|c|}{ Factor name } \\
\hline 1 & Parents' education level \\
\hline 2 & Child's genetic background \\
\hline 3 & Parents' dietary patterns \\
\hline 4 & Parents' physical activity \\
\hline 5 & Medication use \\
\hline 6 & Family income \\
\hline 7 & Frequency of eating \\
\hline
\end{tabular}

The structure of the proposed system will be flexible; after collecting these data, researchers can add more variables as needed. Figure 1 shows the proposed conceptual framework, including the three main components. In the first component, we will use the data collected to train the system to derive the parents' and other related be- 
haviors that have the greatest influence on the children's risk for obesity. We will then build a type-2 fuzzy logic system for predicting the obesity risk for the child, which we expect will provide more-accurate results than with a process prior theoretical framework,

After the completion of this system, we will use it in the intervention process when predicting children's risk for obesity. The demonstration will use the extracted rules to get the best behavior from previous data and allow parents to observe the variables they must change to help their children reduce their obesity risk. This intervention will be applied to the type-2 fuzzy logic system. This system has an interval and general type, and we rely on it because of its ability to make accurate predictions even when faced with high levels of uncertainty.

\subsection{Validity and reliability of the study}

Reliability and validity are critical elements of research. Although they are often used interchangeably, they have different but related meanings. The validity of this study is guaranteed by the large sample size. We plan to include approximately one thousand families in Saudi Arabia. The conceptual design of this study and the experimental design that we will adopt are accurate. It will help gather data that will be generalized to the entire population. The findings will reflect the phenomena being studied and measured. The conceptual model based on type-2 fuzzy logic theory for predicting childhood obesity risk is a tested framework that will help obtain valid data. We will be guided by the conceptual model and the data collection method and analysis to reduce biases. Considering that we will focus on quantitative data, subjective experiences and interpretations will be kept at bay. The reliability of a study entails the degree to which the methodology produces consistent and stable results. Study results can only be valid when the research method is reliable. We believe that by using the correct conceptual and experimental design, the outcome will be repeatable when the study is carried out at a different time under similar conditions. Overall, the validity and reliability of the study will be enhanced by being conscious of biases that may occur and by maintaining integrity in the entire process.

\subsection{Ethical consideration}

All studies must comply with ethical requirements. Considering that we will involve human subjects, no one will be coerced to participate in the study. The participants will have the right to drop out of the study anytime they deem fit. To obtain full consent, we will inform the subjects about the purpose of the study. We will furnish them with adequate information and give them assurances about their participation in the study [38]. We will ensure that they understand the implications of the study and their involvement in it. Doing so will help ensure that the families make independent decisions about whether or not to participate without being subjected to any coercion or pressure. We will endeavor to provide full and accurate information to avoid any deception about the research goals and objectives. We will protect the privacy of the participants by ensuring that we do not use any personally identifiable information 
and will also maintain the anonymity of the participants and safeguard their responses from being accessed by unauthorized persons [37]. We will keep the information on a password-protected computer and will also maintain data integrity by acknowledging all authors who will be used in any section of this paper. We will also analyze the information as it is gathered without any alterations or distortions. Above all, we will protect participating families from any harm, either physical or psychological, by avoiding the use of discriminatory, offensive, or unacceptable language. We will be sensitive to the participant's feelings and emotions, especially those who already have children afflicted with obesity.

\section{Conclusion}

Obesity is one of the world's fastest evolving health challenges in the poorest and wealthiest countries. Childhood obesity is rising exponentially worldwide due to several factors. Family's dirty habits patterns and characteristics play a significant role in causing the risk of obesity for their children. This paper reviewed the Artificial Intelligence techniques used out for the childhood obesity field. Besides, this paper proposed conceptual frameworks that aims to use novel type-2 fuzzy logic methodology that are capable of predicting the risk of obesity for children based on their family's dirty habits patterns, characteristics, and other parameters related to the home environment and the child itself. This prediction will be used as an intervention factor to remediate obesity, which will enhance public health and reduce the costs of later treatments for several obesity-related diseases. We are planning to evaluate the proposed conceptual methodology on at least 1,000 families and their children within Saudi Arabia. The proposed type-2 fuzzy logic-based systems will be capable of handling the encountered uncertainties to achieve better modeling and a more accurate risk of obesity for children. They can also encode the extracted rules in comprehensive ways to provide insights for the best obesity prevention behaviors for getting obese child risk.

\section{$6 \quad$ References}

[1] Anderson PM, Butcher KF, Schanzenbach DW. Understanding recent trends in childhood obesity in the United States. Econ Hum Biol. 2019:1-10. doi:10.1016/j.ehb.2019.02.002

[2] Anderson, P. M., \& Butcher, K. F. (2006). Childhood obesity: Trends and potential causes. The Future of Children, 16(1), 19-45.

[3] Biro FM, Wien M. Childhood obesity and adult morbidities. Am J Clin Nutr 2010; 91 : 1499S-1505S. https://doi.org/10.3945/ajcn.2010.28701b

[4] Pulgaron ER (2013) Childhood obesity: a review of increased risk for physical and psychological comorbidities. Clin Ther 35, 014.

[5] Waters E, de Silva-Sanigorski A, Hall BJ, Brown T, Campbell KJ, Gao Y, Armstrong R, Prosser L, Summerbell CD: Interventions for preventing obesity in children. Cochrane Database Syst Rev. 2011, 12: CD001871. https://doi.org/10.1002/14651858.cd001871.pub3 
[6] Freedman DS, Dietz WH, Srinavisian SR, Berenson GS. The relation of overweight to cardiovascular risk factors among children and adolescents: the Bogalusa Heart Study. Pediatrics1999;103: 1175-1182 .https://doi.org/10.1542/peds.103.6.1175

[7] Waters E, Silva-Sanigorski A, Burford BJ et al (2014) Interventions for preventing obesity in children. Sao Paulo Med J 132(2):128-129. https://doi.org/10.1590/1516-3180.2014132 $\underline{2 \mathrm{t} 2}$

[8] Summerbell CD, Waters, E, Edmunds, LD, Kelly, S, Brown, T, and Campbell, KJ, Interventions for preventing obesity in children. Cochrane database of systematic reviews (Online) 2005; CD001871. https://doi.org/10.1002/14651858.cd001871.pub2

[9] Birch L, Ventura A. Preventing childhood obesity: what works? Int J Obes 2009; 33: S74S81. https://doi.org/10.1038/ijo.2009.22

[10] Bluford DA, Sherry B, Scanlon KS. Interventions to prevent or treat obesity in preschool children: a review of evaluated programs. Obesity (Silver Spring) 2007; 15: 1356-1372. https://doi.org/10.1038/oby.2007.163

[11] Marshall T, Champagne-Langabeer T, Castelli D, Hoelscher D. Cognitive computing and eScience in health and life science research: artificial intelligence and obesity intervention programs. Health Inf Sci Syst. 2017; 5(1):13. https://doi.org/10.1007/s13755-017-0030-0.

[12] Hey T, Tansley S, Tolle KM, editors. The fourth paradigm: data intensive scientific discovery. Jim Grey, foreword, XVII, Microsoft Research; 2009.

[13] Das R. Five technologies that will disrupt healthcare by 2020. Forbes.com, 30 Mar 2016.

[14] Bruna Marmett, Roseana Böek Carvalhoa, Melissa Santos Fortesb, Sílvio César Cazellab, "Artificial Intelligence technologies to manage obesity", Vittalle - Revista de Ciências da Saúde, vol. 30, no. 2, pp. 73-79, 2018. https://doi.org/10.14295/vittalle.v30i2.7654

[15] Valavanis IK, Mougiakakou SG, Marinos S, Karkalis G, Grimaldi KA, Gill R, et al. Gene - Nutrition Interactions in the Onset of Obesity as Cardiovascular Disease Risk Factor based on a Computational Intelligence Method. 8th IEEE International Conference on Bioinformatics and Bioengineering, Vols 1 and 2. 2008:170-5. https://doi.org/10.1109/bibe.20 $\underline{08.4696678}$

[16] Saddek B, Bounechada M, Djoudi A, Harzallah D, Alleg F, Benamrani H. Prevention of Obesity using Artificial Intelligence Techniques. International Journal of Science and Engineering Investigations 2012; 1(9):146-50.

[17] Cruz MR, Martins C, Dias J, Pinto JS. A validation of an intelligent decision-making support system for the nutrition diagnosis of bariatric surgery patients. JMIR Med Inform 2014; 2(2):e8. https://doi.org/10.2196/medinform.2984

[18] Lopez-Meyer P, Schuckers S, Makeyev O, Sazonov E. Detection of periods of food intake using Support Vector Machines. Conf Proc IEEE Eng Med Biol Soc 2010; 1004-7. https:// doi.org/10.1109/iembs.2010.5627796

[19] O. Babajide et al., "Application of Unsupervised Learning in Weight-Loss Categorisation for Weight Management Programs," 2019 10th International Conference on Dependable Systems, Services and Technologies (DESSERT), Leeds, United Kingdom, 2019, pp. 94101. https://doi.org/10.1109/dessert.2019.8770032

[20] Muhamad Adnan M, Husain W, Damanhoori F. A survey on utilization of data mining for childhood obesity prediction. Information and Telecommunication Technologies (APSITT) 2010; 1-6.

[21] Novak B, Bigec M. Childhood obesity prediction with artificial neural networks. Computer-Based Medical Systems 1996; 77-82. https://doi.org/10.110 9/cbms.1996.507129 
[22] Novak B, Bigec M. Application of artificial neural networks for childhood obesity prediction. Artificial Neural Networks and Expert Systems 1995; 377380. https://doi.org/10.1109/annes.1995.499512

[23] Adnan MHBM, Husain W, Rashid N. Parameter Identification and Selection for Childhood Obesity Prediction Using Data Mining. 2nd International Conference on Management and Artificial Intelligence 2012.

[24] Adnan M, Hariz M, Husain W, Rashid A. A hybrid approach using Naïve Bayes and Genetic Algorithm for childhood obesity prediction. Computer \& Information Science (ICCIS) 2012; 281-285. https://doi.org/10.1109/iccisci.2012.6297254

[25] Zhang S, Tjortjis C, Zeng X, Qiao H, Buchan I, Keane J. Comparing data mining methods with logistic regression in childhood obesity prediction. Information Systems Frontiers 2009; 11: 449-460. https://doi.org/10.1007/s10796-009-9157-0

[26] Dugan, T., Mukhopadhyay, S., Carroll, A., Downs, S. (2015) Machine Learning Techniques for Prediction of Early Childhood Obesity. Applied Clinical Informatics, 6(3),pp.506-520. https://doi.org/10.4338/aci-2015-03-ra-0036

[27] Ergun U. The classification of obesity disease in logistic regression and neural network methods. J Med Syst 2009; 33(1): 67-72. https://doi.org/10.1007/s10916-008-9165-5

[28] Disse E et al. An artificial neural network to predict resting energy expenditure in obesity. Clin Nutr 2017; Sep 1. pii: S0261-5614(17)30258-3.

[29] Valavanis IK et al. A multifactorial analysis of obesity as CVD risk factor: use of neural network based methods in a nutrigenetics context. BMC Bioinformatics 2010; 11: 453. https://doi.org/10.1186/1471-2105-11-453

[30] Predicting overweight and obesity in adulthood from body mass index values in childhood and adolescenc

[31] R. Stathacopoulou, M. Grigoriadou, M. Samarakou, and D. Mitropoulos, "Monitoring students' actions and using teachers' expertise in implementing and evaluating the neural network-based fuzzy diagnostic model," Expert Systems with Applications, vol. 32, no. 4, pp. 955-975, May 2007. https://doi.org/10.1016/j.eswa.2006.02.023

[32] K. Almohammadi, H. Hagras, D.Alghazzawi, G. Aldabbagh "A survey of the Artificial Intelligence Techniques Employed for Adaptive Educational Systems," Journal of Artificial Intelligence and Soft Computing Research, 2016. https://doi.org/10.1515/jaiscr-2017-0004

[33] J. M. Mendel, "Uncertain Rule-Based Fuzzy Logic Systems," Prentice Hall 2001.

[34] J. M. Mendel, H. Hagras, W. Tan, W. Melek, and H. Ying, "Introduction to Type-2 Fuzzy Logic Control," John Wiley and IEEE Press, Hoboken, NJ, 2014. https://doi.org/10.1002/ $\underline{9781118886540}$

[35] Rautianen, I.,\& Ayramo, S. (2019). Predicting overweight and obesity in later life from childhood data. A review of predictive modelling approaches

[36] Sahoo K, Sahoo B, Choudhury AK, et al. Childhood obesity: causes and consequences. J Family Med Prim Care 2015;4(2):187-92. https://doi.org/10.4103/2249-4863.154628

[37] Anderson PM, Butcher KE. Childhood obesity: Trends and potential causes. Future Child. 2006; 16:19-45.

[38] Sanjari, M., Bahramnezhad, F., Fomani, F.K., Shoghi, M., Cheraghi, M.A. (2014). Ethical challenges of researchers in qualitative studies: The necessity to develop a specific guideline. Journal of Medical Ethics Hist Med, 7, 14 


\section{$7 \quad$ Author}

Khalid Almohammadi completed his bachelor's degree in computer science from Taibah University in the Kingdom of Saudi Arabia. He then pursued a profession in teaching at Tabuk University. Building on this experience, he was awarded a scholarship to complete his master's in computer science (MSc) at Newcastle University in England, which he obtained with distinction in 2011. He recently completed his $\mathrm{PhD}$ in computer science from Essex University in England, which enables him to incorporate all his previous knowledge and experience in education and e-learning. The focus of his research is the development of theoretical and practical environments to enhance students' learning experience and engagement by using fuzzy logic systems. He currently works as an assistant professor in the Computer Science Department at Community college Tabuk University.

Article submitted 2019-12-12. Resubmitted 2020-01-10. Final acceptance 2020-01-10. Final version published as submitted by the authors. 